\title{
CONSIDÉRATIONS
}

\section{SUR LE GENRE ANOPLOCEPHALA}

PAR

JEAN G. BAER

Travail du Laboratoire de Zoologie de l'Université de Neuchâtel

(AVEC DEUX PLANCHES)

$\mathrm{Au}$ cours de certaines recherches entreprises sur la structure des Cestodes, nous avons eu à nous occuper plus spécialement de la sous-famille des Anoplocephalinae, et en particulier du genre Anoplocephala.

Le manque d'homogénéité, ainsi que la diversité d'anatomie de différents groupes d'espèces de ce genre, nous ont fourni le point de départ de cette étude, qui fut facilitée par le fait d'avoir à notre disposition du matériel et des préparations des principales espèces.

Nous tenons à exprimer ici toute notre reconnaissance à notre maître et professeur, M. le $D^{r}$ O. Fuhrmann, qui a mis son matériel à notre disposition et qui n'a cessé de nous donner de précieux conseils.

Le genre Anoplocephala fut créé en 1848 par E. BLANCHARD, avec $A$. perfoliata (Gœze) comme espèce type. Depuis lors, il a été décrit, plus ou moins bien, 38 espèces qui devraient, d'après leurs auteurs, rentrer dans ce genre. Cependant, au cours de recherches ultérieures, plusieurs de ces espèces ont trouvé leur place dans d'autres genres déjà connus, et certaines dans des genres nouveaux ; il en résulte qu'actuellement le genre Anoplocephala comprend 20 espèces, dont seules une dizaine sont bien connues. 
En étudiant de plus près ces 20 espèces, nous avons été frappé par trois points importants, à savoir :

1. Dans la répartition des testicules, on peut distinguer deux types; l'un où les testicules occupent invariablement toute la largeur du parenchyme médullaire, et l'autre où les testicules sont franchement antiporaux, pouvant même dépasser le nerf longitudinal antiporal.

2. Il y a également deux types distincts de système excréteur, l'un ne présentant pas de particularités, composé de deux vaisseaux longitudinaux dorsaux et de deux vaisseaux ventraux, ces derniers réunis entre eux dans la partie postérieure de chaque segment par un vaisseau transversal, les vaisseaux dorsaux étant en dessus ou en dehors des vaisseaux ventraux. Le deuxième type est celui d'un système excréteur rétiforme, les vaisseaux longitudinaux étant reliés entre eux par un système de vaisseaux secondaires dont le diamètre est égal à celui des vaisseaux principaux, le tout formant un réseau extrêmement compliqué.

3. Deux types d'ovaires sont également à distinguer; l'un où l'ovaire est poral ou médian, ayant la forme d'un éventail, et l'autre où l'ovaire est formé de deux ailes, une aile porale et une aile antiporale plus grande. Ces deux ailes occupent presque toute la largeur du parenchyme médullaire et portent des diverticules en forme de massue partant d'un cordon cellulaire transversal.

Ces trois points nous paraissent d'une importance suffisante pour justifier la création de deux genres. Dans la suite, nous donnerons avec la diagnose de chaque genre la liste des espèces y appartenant, ainsi que leur synonymie. Les espèces marquées par un astérisque ont été étudiées par nousmême.

\section{Genre Anoplocephala E. Blanchard e. p. Baer.}

Anoplocéphaliens à segments toujours plus larges que longs ; pores génitaux unilatéraux ; conduits sexuels dorsaux par rapport au nerf et aux vaisseaux longitudinaux ; vésicule séminale externe présente ; vaisseaux excréteurs longitudinaux reliés entre eux par un système compliqué de vaisseaux secondaires, donnant au tout un aspect rétiforme ; testicules répartis sur toute la largeur du parenchyme médullaire; ovaire occupant presque toute la largeur du parenchyme médullaire, formé de deux ailes, une porale et l'autre antiporale, plus grande ; glande vitellogène porale ; vagin situé 
en dessous de la poche du cirre; utérus en forme de tube transversal, devenant sacciforme et lobé par évaginations successives de ses parois; œufs munis d'un appareil pyriforme bien développé.

Adultes chez Perissodactyla, et plus rarement chez Hyracoidea et Proboscidea.

Type : Anoplocephala perfoliata (Gœze).

Dans le genre Anoplocephala ainsi conçu rentrent les espèces suivantes :

\section{* Anoplocephala perfoliata (Goeze).}

Synonymie : Taenia perfoliata. Gœze. 1782.

Taenia equina. Chabert. 1782.

Halysis perfoliata. Zeder. 1803.

Alyselminthus perfoliata. Zeder. 1828.

Anoploce phala perfoliata. E. Blanchard. 1848.

Hôtes: Equus caballus. E. asinus.

* Anoplocephala perfoliata (Goeze), var. zebrae (Collin).

Synonymie : Taenia zebrae. Collin. 1891.

Anoplocephala zebrae. Fuhrmann. 1910.

Anoplocephala rhodesiensis. Southwell. 1921.

Hôte : Equus burchelli.

Nous avons pu faire, grâce à un matériel abondant, une étude comparée de $A$. perfoliata (Gœze) du cheval et de A. rhodesiensis Southwell du zèbre. Nous ne trouvons aucun caractère anatomique constant qui puisse justifier deux espèces distinctes.

Southwell (31) admet deux caractères constants: 1) A. rhodesiensis est plus massif et comporte environ deux fois plus de segments que $A$. perfoliata. Ce caractère purement morphologique ne peut justifier à notre avis une espèce distincte, mais tout au plus une variété. 2) La moitié postérieure de $A$. rhodesiensis est entièrement stérile, tandis que chez A. perfoliata, les segments deviennent de plus en plus mûrs. Ce caractère non plus n'est pas constant, car KAHANE (15) signale des cas où la moitié postérieure de $A$. perfoliata était stérile; Bischoff (1) signale le même phénomène chez A. spatula (v. Linstow).

Nous proposons pour cette variété le nom de $A$. perfoliata (Gœze) var. zebrae (Collin). 
* Anoplocephala magna (Abilgaard).

Synonymie : Taenia magna. Abilgaard. 1789.

Taenia equi. Müller. 1780. Ex parte.

Taenia equina. Pallas. 1781. Ex parte.

Alyselminthus plicatus. Zeder. 1800.

Halysis plicata. Zeder. 1803.

Taenia zebrae. Rudolphi. 1808.

Taenia plicata. Rudolphi. 1810.

Anoplocephala zebrae. Railliet. 1891.

Anoplocephala restricta. Railliet. 1893.

Anoplocephala magna var. pediculata. Railliet. 1893.

Anoplocephala magna var. strangulata. Railliet. 1893.

Anoplocephala plicata var. restricta. Railliet. 1895.

Anoplocephala magna. Spengel. 1905.

Anoplocephala plicata var. servei. Bounhiol. 1912.

Hôtes: Equus caballus. E. asinus. E. burchelli.

* Anoplocephala magna (Abilgaard) var. gigantea (Peters).

Synonymie : Taenia gigantea. Peters. 1856.

Taenia magna. Murie. 1870.

Plagiotaenia gigantea. Peters. 1871.

Taenia (Anoplocephala) gigantea. Braun. 1895. Anoplocephala latissima. Deiner. 1912.

Schitzotaenia latissima. Douthitt. 1915.

Schitzotaenia gigantea. Douthitt. 1915.

Anoplocephala vulgaris. Southwell. 1921.

Hôtes : Rhinoceros indicus. R. sondaicus. R. bicornis.

Ce Taenia vraiment gigantesque a été étudié par plusieurs auteurs $(4,5,11,19,21,22,26)$. Parmi les plus récents,

\section{Planche I}

Anoplocephala magna (Abilgaard).

Fig. 1. Coupe horizontale montrant l'aile porale de l'ovaire (ov), le réceptacle séminal (rs) et la glande vitellogène (gv). Le système excréteur se présente sous forme de trous (ex).

Fig. 2. Coupe transversale montrant la position relative des testicules (T) et de l'ovaire.

Fig. 3. Coupe horizontale montrant la disposition des testicules. 
Fig. 1

Fig. 2
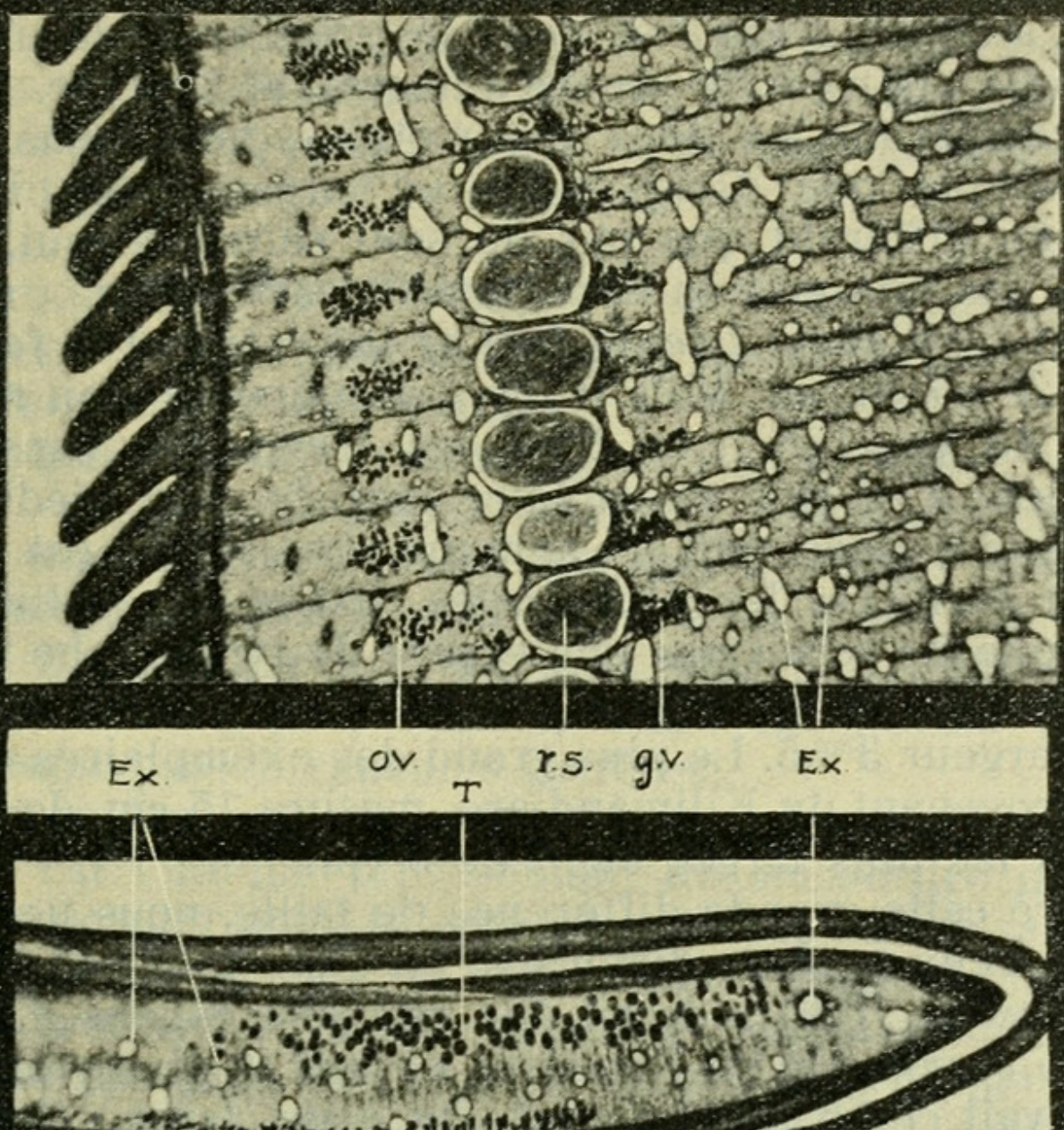

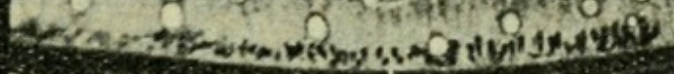

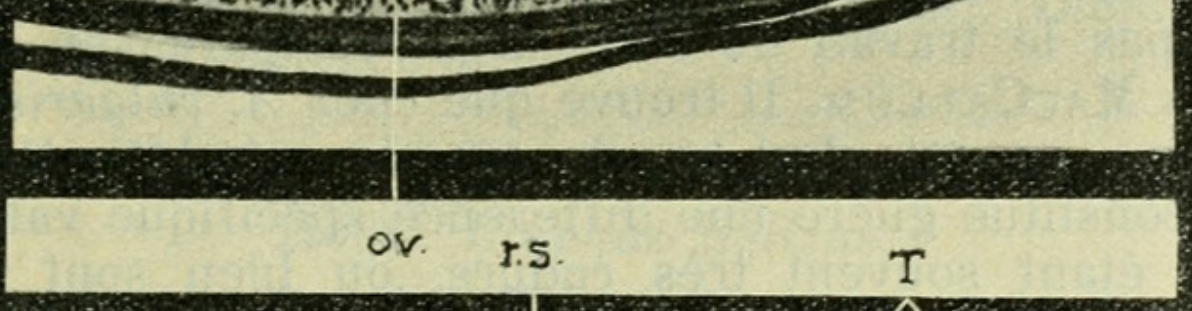

Fig. 3

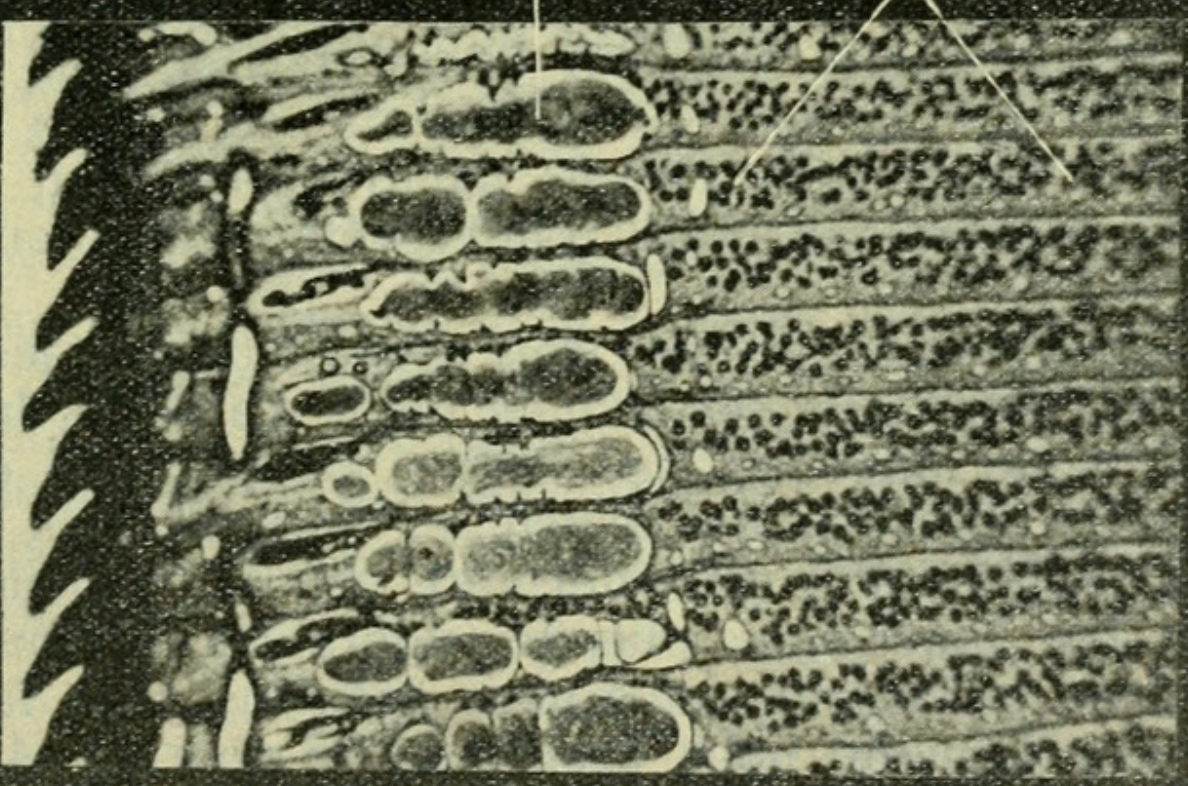


citons d'abord les travaux des MacCallum (19) et de Deiner (4), ces auteurs ayant fait pour la première fois une étude anatomique approfondie de cet intéressant Cestode.

En comparant les deux descriptions, nous nous sommes rendu compte que nous étions en présence de la même espèce, ne différant que par un seul caractère qui, à notre avis, n'a pas une grande importance: les MACCALLUM disent: ... At that time about twenty feet of the worm was found and some of it preserved. Mais il n'y a absolument rien nous permettant de conclure que tous ces fragments, sans scolex, provenaient d'un seul exemplaire long de vingt pieds $(6 \mathrm{~m}$.)! La largeur donnée par les auteurs américains est de $7^{\mathrm{cm}}, 5$ pour les segments les plus larges. Est-ce que cette mesure gigantesque ne serait pas une erreur de typographe? Cependant DeINer donne comme longueur maximale $10 \mathrm{~cm}$. et comme largeur $3^{\mathrm{cm}}, 5$. Le plus grand des exemplaires que nous ayons, provenant du Kilimandjaro, mesure $15 \mathrm{~cm}$. de long, les segments les plus larges étant de $3 \mathrm{~cm}$.

Malgré cette grande différence de taille, nous ne pouvons admettre deux espèces distinctes.

Southwell (26) a décrit une nouvelle espèce $A$. vulgaris chez $R$. bicornis, tué en Rhodésie, et dont le plus grand exemplaire avait $7^{\mathrm{cm}}, 5$ de long et $2^{\mathrm{cm}}, 7$ de large. Cet auteur ne cite pas le travail de DEINER et compare son espèce à celle des MACCALlum. Il trouve que chez $A$. vulgaris le cirre est inerme, ce qui n'est pas le cas chez A. latissima: mais ceci ne constitue guère une différence spécifique valable, les crochets étant souvent très caducs, ou bien sont arrachés après la copulation. De plus, il admet les données des MACCALLUM disant que la longueur totale était de plus de vingt pieds, tandis qu'il estime la longueur de son espèce à $12 \mathrm{~cm}$. seulement. Comme nous l'avons montré plus haut, nous ne pouvons accepter cette façon d'interpréter les auteurs américains.

Nous considérons donc ces trois espèces déjà citées comme étant toutes identiques, l'anatomie étant d'ailleurs la même. De plus, cette anatomie ne diffère en rien de celle de A. magna (Abilgaard) du cheval, et nous serions presque enclins à l'assimiler à cette dernière espèce. Cependant, vu l'hôte, et les dimensions plus considérables, surtout en ce qui concerne la largeur, nous nous décidons à en faire une variété pour laquelle nous proposons le nom de $A$. magna (Abilgaard) var. gigantea (Peters).

Douthitt (5) place l'espèce des MacCallum et celle de 
DEINER dans le genre Schitzotaenia Janicki, mais nous ne pouvons admettre cette manière de voir, vu que l'utérus de ce genre est primitivement réticulé, ce qui n'est pas le cas pour l'espèce en question, quoi qu'en dise l'auteur, qui n'a cependant pas examiné le matériel.

\section{Anoplocephala spatula (v. Linstow).}

Synonymie : Taenia (Anoplocephala) spatula v. Linstow. 1901. Hôte : Heterohyrax mossambica.

Ce Taenia a été trouvé pour la première fois par v. Linstow (18), en Afrique orientale allemande, chez Heterohyrax mossambica. JANICKI (cit. 24) a voulu l'assimiler au genre Inermicapsifer Janicki, mais à la suite des observations de Bischoff (1), il ne peut plus être question de le placer dans ce genre, l'utérus étant sacciforme et ne se décomposant pas en capsules ovifères.

Il est intéressant de constater que le genre Hyrax ou Procavia est l'unique représentant du sous-ordre des Hyracoidea, ce dernier étant intermédiaire entre les Perissodactyla et les Proboscidea chez un représentant desquels il a également été trouvé un Taenia du genre Anoplocephala, et dont nous parlerons plus bas. Il nous semble qu'il ne peut y avoir d'hésitation à placer cette espèce dans le genre Anoplocephala tel que nous le comprenons.

\section{Anoplocephala manubriata Railliet, Henry et Bauche. 1914.}

Hôte: Elephas indicus.

Les auteurs placent cette espèce tout près de l'espèce précédente. Cependant, elle présente une particularité que nous n'avons pas encore rencontrée dans le genre Anoplocephala, à savoir que les deux ailes de l'ovaire sont d'égale grandeur et que ce dernier organe est médian. Ces deux caractères rapprochent $A$. manubriata du nouveau genre que nous avons créé ; cependant le système excréteur et la position des testicules sont ceux du genre Anoplocephala, de sorte que nous sommes amenés à considérer $A$. manubriata comme étant une espèce marquant le passage d'un genre à l'autre; pour le moment, nous la maintenons dans le genre Anoplocephala. 
Genre Anoplocephaloides. n. gen.

Anoplocéphaliens à segments toujours plus larges que longs; pores génitaux unilatéraux; conduits sexuels dorsaux, par rapport au nerf et aux vaisseaux longitudinaux; vésicule séminale externe présente; système excréteur non réticulé ; testicules du côté antiporal pouvant, dans certains cas, dépasser latéralement le nerf longitudinal ; ovaire poral en forme d'éventail ; vagin situé ventralement à la poche du cirre ; utérus en forme de tube transversal, devenant sacciforme et lobé par évaginations successives de ses parois; œufs munis d'un appareil pyriforme bien développé.

Adultes chez Rodentia et dans un cas chez Perissodactyla.

Type: Anoplocephaloides infrequens (Douthitt).

Nous avons choisi comme espèce type celle qui est le mieux étudiée et dont le matériel est le plus abondant, plutôt que de prendre l'espèce la plus ancienne dont fort peu d'exemplaires sont connus.

Le genre Anoplocephaloides comprend les espèces suivantes :

Anoplocephaloides infrequens (Douthitt).

Synonymie: Anoplocephala infrequens. Douthitt. 1915.

Hôtes: Geomys bursarius. Microtus sp.

Anoplocephaloides variabilis (Douthitt).

Synonymie : Anoplocephala variabilis. Douthitt. 1915. Hôte : Geomys bursarius.

* Anoplocephaloides wimerosa (Moniez).

Synonymie: Taenia wimerosa. Moniez. 1880. Anoplocephala wimerosa. R. Blanchard. 1891. Andrya wimerosa. Railliet. 1893. Anoplocephala wimerosa. Douthitt. 1915.

Hôtes: Lepus cuniculus. L. variabilis.

\section{Planche 2}

Fig. 4. Coupe horizontale de Anoplocephaloides mamillana (Mehlis) montrant la situation et la structure de l'ovaire.

Fig. 5. Coupe horizontale de Anoplocephaloides mamillana (Mehlis) montrant l'aspect des testicules et de la poche du cirre (pc). Dans le bas de la photo on voit un cirre dévaginé (c).

Fig. 6. Coupe horizontale de Anoplocephala magna (Abilgaard) montrant la structure et la situation de l'ovaire. 
Fig. 4

Fig. 5
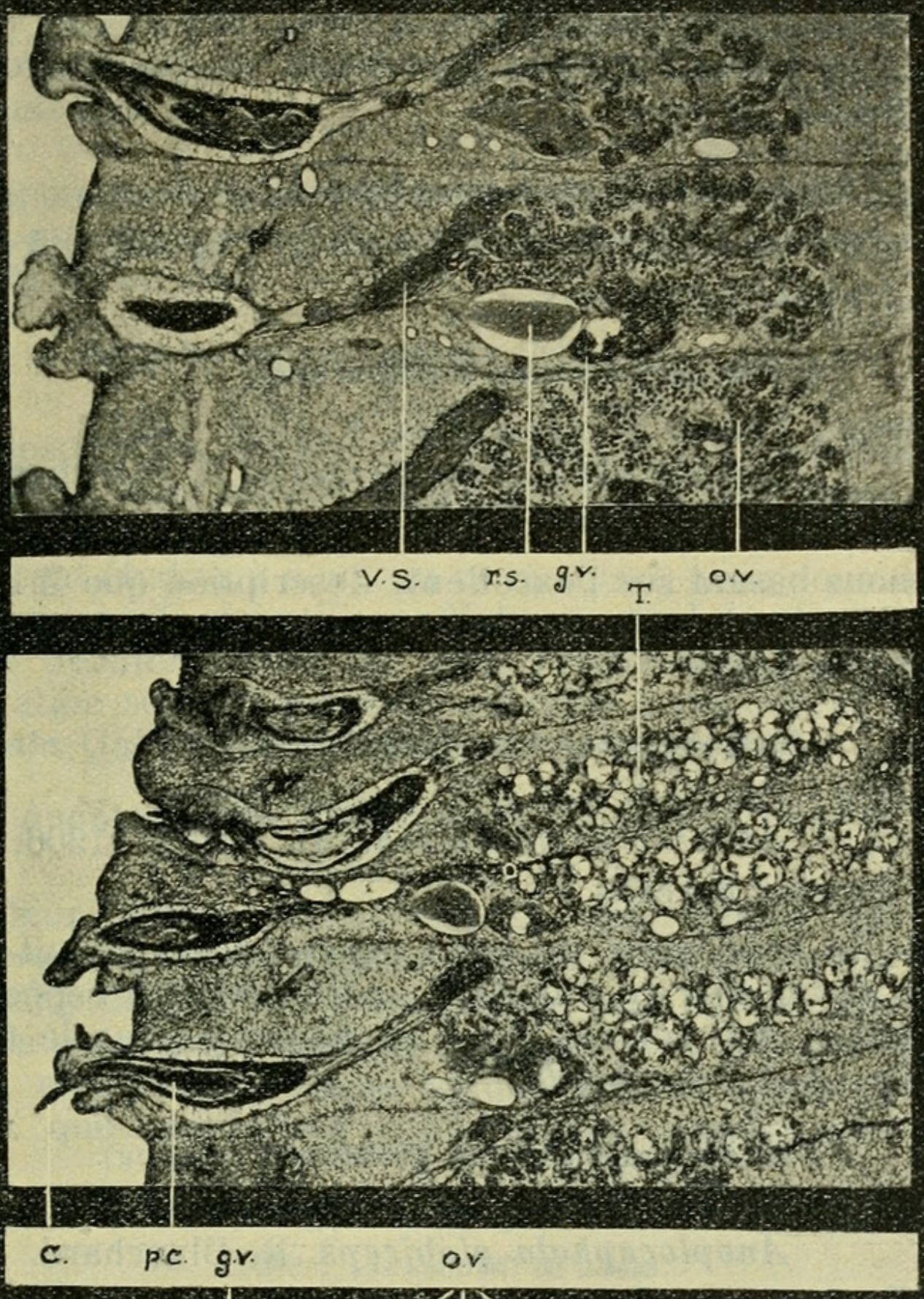

Fig. 6

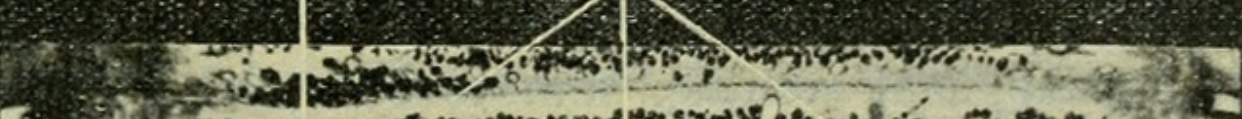

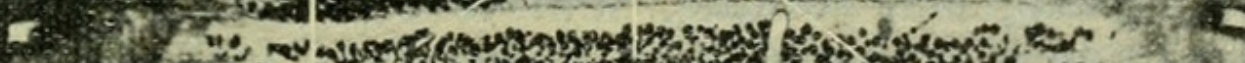

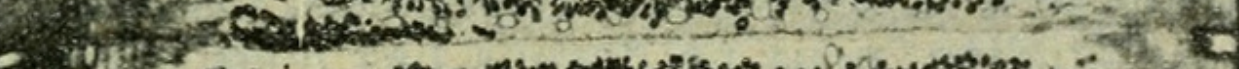

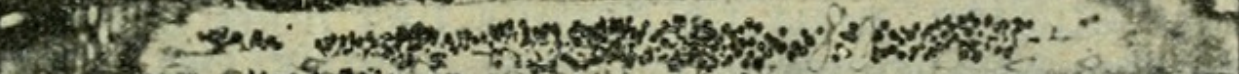
cuncos o

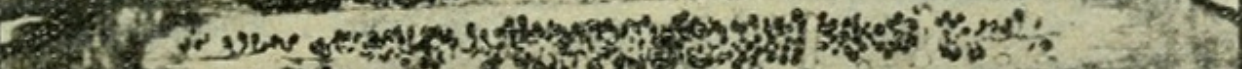

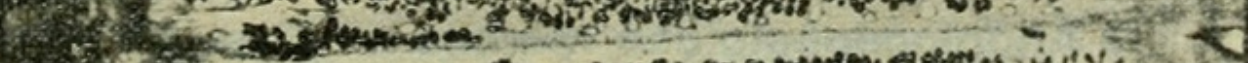

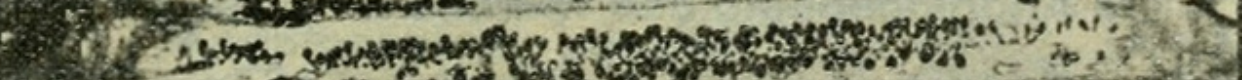

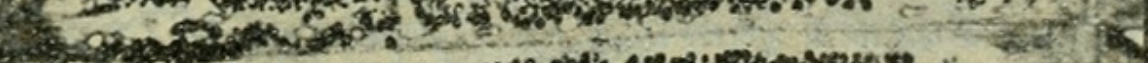

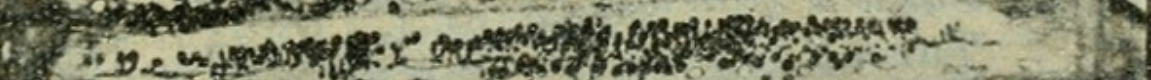

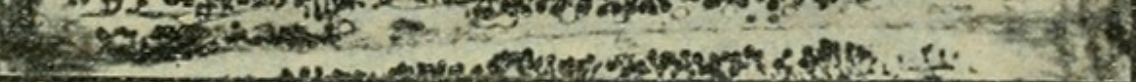


* Anoplocephaloides mamillana (Mehlis).

Synonymie : Taenia mamillana. Mehlis. 1831.

Anoplocephala mamillana. R. Blanchard. 1891.

Hôte : Equus caballus.

Anoplocephaloides campestris (Cholodkovsky).

Synonymie : Anoplccephala campestris. Cholodkovsky. 1912. Hôte : Arvicola campestris.

Anoplocephaloides transversaria (Krabbe).

Synonymie : Taenia transversaria. Krabbe. 1879.

Anoploce phala iransversaria. R. Blanchard. 1891.

Hôte : Arctomys marmota.

En nous basant sur l'excellente description que ZschoкKE (32) a donnée de cette espèce, nous la plaçons sans hésitation dans ce genre.

\section{Anoplocephaloides blanchardi (Moniez).}

Synonymie : Taenia blanchardi. Moniez. 1891.

Anoplocephala blanchardi. Janicki. 1906.

Hôte : Arvicola agrestis.

Dans la synonymie de cette espèce devrait peut-être se trouver $A$. inermis (v. Linstow); cette dernière espèce étant cependant si mal connue, nous ne pouvons pour le moment l'assimiler à $A$. blanchardi.

? Anoplocephaloides globiceps (Diesing).

Synonymie : Taenia globiceps. Diesing. 1856. Anoplocephala globiceps. R. Blanchard. 1891.

Hôte : Tapirus americanus.

Cette espèce, mal connue, semble être très voisine, si ce n'est identique à $A$. mamillana. Cependant, jusqu'à ce qu'elle soit mieux étudiée, nous ne devons pas nous prononcer sur sa position systématique.

? Anoplocephaloides inermis (v. Linstow).

Synonymie : Taenia inermis. v. Linstow. 1878.

Taenia arvicolae. R. Blanchard. 1891.

Anoplocephala arvicolae. Janicki. 1906.

Anoplocephala inermis. Douthitt. 1915.

Hôte : Arvicola campestris. 
JANicki (14), se basant sur la description de v. Linstow, fait rentrer cette espèce dans le genre Anoplocephala. Cependant, la description étant tout à fait insuffisante, nous ne mettons cette espèce que provisoirement dans le genre Anoplocephaloides.

Occupons-nous maintenant des espèces du genre Anoplocephala qui ont été insuffisamment décrites, ou bien qui ont été mises à tort dans ce genre.

Anoplocephala dentata Galli-Valerio. 1905.

Hôte : Arvicola nivialis.

La description donnée par cet auteur (10) est tout à fait insuffisante et ne permet même pas d'assimiler cette espèce au genre Anoplocephala.

Désirant étudier à nouveau ce matériel, nous nous sommes adressé au professeur GALLI-VALERIO, à Lausanne ; cependant, ce dernier refusa de nous l'envoyer! Ce refus à la collaboration scientifique nous entraîne à considérer l'espèce A. dentata Galli-Valerio comme un nomen nudum.

Anoplocephala globocephala, MacCallum. 1921?!

Hôte : Raja sp.

L'auteur décrit sommairement une espèce du genre Anoplocephala, trouvé dans un poisson !! Le dessin qui accompagne cette description nous montre un Cestode dont l'anatomie n'a absolument rien de commun avec celle de Anoplocephala, elle semble même unique en son genre. Nous ne pouvons que considérer cette espèce comme un nomen nudum.

Anoplocephala sp. Janicki. 1904.

Hôte : Arvicola arvalis.

Jusqu'à ce qu'une étude approfondie ait été faite de cette espèce, il n'est pas possible de lui assigner une position systématique.

Viennent en dernier lieu les deux seules espèces qui aient été trouvées chez des oiseaux.

Anoplocephala minima Mello. 1912.

Hôte: Phasianus dom.

D'après Fuhrmann $(9,10)$, cette espèce, fort mal décrite, n'est pas un Anoplocephala, la description ne permettant même pas de l'attribuer à la famille des Anoplocéphalides. 


\section{$-14-$}

* Anoplocephala pinguis Fuhrmann.

Synonymie: Bertia (Bertiella) pinguis. Fuhrmann. 1902. Anoplocephala pinguis. Fuhrmann. 1921.

Hôte : Bucorax abyssinicus.

En examinant les préparations originales, nous avons pu nous rendre compte que cette espèce intéressante présente bien des analogies avec les genres Bertiella et Anoplocephala, sans cependant pouvoir être attribuée définitivement à l'un d'eux; néanmoins, il nous semble que cette espèce tend plutôt vers le genre Bertiella que vers le genre Anoplocephala, c'est pourquoi nous nous proposons de l'attribuer de nouveau à ce genre.

\section{Anoplocephala omphalodes (Hermann).}

Hôtes: Mus arvalis. M. amphibius.

Nous jugeons bon de signaler ici que cette espèce a été choisie par LüHE (17) comme espèce type d'un nouveau genre, Paranoplocephala Lühe. 1910.

En résumé, nous avons divisé le genre Anoplocephala E. Blanchard en deux genres :

1. Anoplocephala E. Blanchard, e. p. Baer.

2. Anoplocephaloides n. gen.

Ces deux genres comprennent les espèces suivantes :

Genre Anoplocephala E. Blanchard, e. p. Baer.

A. perfoliata (Gœze).

A. perfoliata (Gœze) var. zebrae (Collin).

A. magna (Abilgaard).

A. magna (Abilgaard) var. gigantea (Peters).

A. spatula (v. Linstow).

A. manubriata. Railliet, Henry et Bauche.

Genre Anoplocephaloides n. gen.

A. infrequens (Douthitt).

A. variabilis (Douthitt).

A. wimerosa (Moniez).

A. mamillana (Mehlis).

A. campestris (Cholodkovsky).

A. transversaria (Krabbe).

A. blanchardi (Moniez).

A. globiceps (Diesing).

A. inermis (v. Linstow). 
Espèces incertaines ou non justifiées :
A. dentata Galli-Valerio.
A. globocephala MacCallum.
A. minima Mello.
A. sp. Janicki.

A. pinguis Fuhrmann, a de nouveau été mis dans le genre Beriiella.

\section{BIBLIOGRAPHIE}

(1) Bischoff, C. R. - Cestoden aus Hyrax. Rev. Suisse de Zool. T. 21, 1913.

(2) Bourquin, J. - Cestodes de Mammifères. Le genre Bertia. Rev. Suisse de Zool. T. 13, 1905.

(3) Chоцорколsкy, N. A. - Erklärender Katalog der Sammlung parasitischer Würmer des zoologischen Kabinets der Kais. militär-medizinische Akademie. Lfg. 1. Bandwürmer (Cyclophyllidea). St-Petersburg, 1912.

(4) Deiner, E. - Anatomie der Anoplocephala latissima /nom. nov.). Arb. Zool. Inst. Wien 19. 1912.

(5) Douthitt, H. - Studies on the Cestode family Anoplocephalidae. Illinois Biological Monographs. Vol. 1, № 3, 1915.

(6) Fuhrmann, O. - Die Anoplocephaliden der Vögel. Centralbl. f. Bakt. u. Parasit. Bd. 32, 1902.

(7) Fuhrmann, O. - Cestoden der Schwedischen zoologischen Expedition nach dem Kilimandjaro, dem Meru, und Deutsch-Ostafrika. Stockholm, 1909.

(8) Fuhrmann, O. - Publikationen und Referate. Vermes. Cestodes für 1912, 1913.

(9) Fuhrmans, O. - Einige Anoplocephaliden der Vögel. Centralbl. f. Bakt. u. Parasit. Bd. 87, 1921.

(10) Galli-Valerio, B. - Einige Parasiten von Arvicola nivialis. Zool. Anz. Leipz. Bd. 28, 1905.

(11) Garrod, A. H. - On the Taenia of the Rhinoceros of the Sunderbunds. Proc. Zool. Soc. London, 1877.

(12) Govgh, L. H. - Notes on South-African Parasites. Ann. Rep. S. A. A. A. S., 1908.

(13) Janicki, C. v. - Zur Kenntniss einiger Säugetiercestoden. Zool. Anz. Leipz. Bd. 27, 1904.

(14) JаNicki, C. v. - Studien an Sängetiercestoden. Zeitschr.

f. wiss. Zool. 81, 1906. 
(15) Kahane, Z. - Anatomie von Taenia perfoliata. Goeze. Zeitschr. f. wiss. Zool. 34, 1880.

(16) LüHE, M. - Mitteilungen über wenig bekannte bez. neue südamericanische Taenien des $k$. $k$. naturhistorischen HofMuseum in Wien. Archiv f. Naturg. 61, I, 1895.

(17) LüHe, M. - Cestodes. Heft. 18. Die Süsswasserfauna Deutschlands. Jena, 1910.

(18) Linstow, O. v. - Helminthen von den Ufern des NyassaSees. Jenaische Zeitschr. f. Naturw. Bd. 35, 1901.

(19) MacGallum, G. A. \& MacCallum, W. G. - On the structure of Taenia gigantea. Peters. Zool. Jahrb. Abt. f. Syst. Bd. 32, Heft 5, 1912.

(20) Machallum, G. A. - Studies in Helminthology. Zoopathologica. Vol. 1, No 6, 1921.

(21) Murie, J. - On a probable new species of Taenia from the Rhinoceros. Proc. Zool. Soc. London, 1870.

(22) Peters, W. - Note on the Taenia from the Rhinoceros lately described by $D^{r}$ J. Murie. Proc. Zool. Soc. London, 1871.

(23) Rallliet, A. - Traité de Zoologie médicale et agricole. $2^{\text {me }}$ éd. Paris, 1893.

(24) Railliet, A., Henry, A. \& Bauche, J. - Sur les Helminthes de l'Eléphant d'Asie. Bull. Soc. Path. exot. T. 7, Nos 1, 2, 3. 1914.

(25) Saint-Remy, G. Le développement embryonnaire dans le genre Anoplocephala. Archiv. de Parasit. 3, No 2, 1900.

(26) Southwell, T. - A new species of Cestode /Anoplocephala vulgaris/ from an African Rhinoceros. Ann. Trop. Med. \& Parasit. Liverpool. Vol. 14, No 3, 1921.

(27) Stiles, C. W. - A revision of the adult Tapeworms of Hares and Rabbits. Proc. U. S. Nat. Mus. Vol. 19, No 1105, 1896.

(28) Stiles, C. W. \& Hassal, A. - A Revision of the Adult Cestodes of Cattle, Sheep and allied Animals. U. S. Dept. of Agric. Bull. No 4, 1893.

(29) Stiles, C. W. \& Hassal, A. - Index catalogue of medical and veterinary zoology. Washington, 1902.

(30) Scheibel, A. - Der Bau der Taenia magna. Abilgaard. (T. plicata. Zeder). Dissertation. Giessen, 1895.

(31) Yorke, W. \& Southwell, T. - Lappeted Anoplocephala in Horses. Ann. Trop. Med. \& Parasit. Vol. 15, No 3. Liverpool, 1921.

(32) Zsснокке, F. - Recherches sur la structure anatomique et histologique des Cestodes. Mem. Inst. Nat. Genevois. Genève, 1889.

Manuscrit reçu le 20 septembre 1923.

Dernières épreuves corrigées le 9 novembre 1923. 


\section{$2 \mathrm{BHL}$ Biodiversity Heritage Library}

Baer, Jean G. 1923. "Considérations sur le genre Anoplocephala." Bulletin de la Socie

te

neucha

A

teloise des sciences naturelles 48, 3-16. https://doi.org/10.5962/bhl.part.25604.

View This Item Online: https://www.biodiversitylibrary.org/item/45513

DOI: https://doi.org/10.5962/bhl.part.25604

Permalink: https://www.biodiversitylibrary.org/partpdf/25604

\section{Holding Institution}

American Museum of Natural History Library

\section{Sponsored by}

American Museum of Natural History

\section{Copyright \& Reuse}

Copyright Status: NOT_IN_COPYRIGHT

This document was created from content at the Biodiversity Heritage Library, the world's largest open access digital library for biodiversity literature and archives. Visit BHL at https://www.biodiversitylibrary.org. 